\title{
Shoulder-hand syndrome in cervical spinal cord injury
}

\author{
P S Aisen MD, ${ }^{1}$ M L Aisen $\mathrm{MD}^{2}$ \\ ${ }^{1}$ Department of Medicine, Box 1230, Mount Sinai Medical Center, One Gustave L Levy \\ Place, New York, New York 10029, USA; ${ }^{2}$ The Burke Rehabilitation Center, 785 \\ Mamaroneck Avenue, White Plains, New York 10605, USA.
}

To characterize the occurrence of shoulder-hand syndrome (SHS) complicating the rehabilitation of patients with cervical spinal cord injury, we reviewed the medical records of 43 consecutive patients admitted to the Burke Rehabilitation Center with cervical spinal cord injury, focusing on the clinical features of SHS: shoulder pain, hand/wrist pain, edema, vasomotor changes, trophic changes and osteoporosis on x-ray. Twenty-seven patients $(63 \%)$ had three or more features of SHS. The number of features correlated with age $(r=0.495, p=0.0007)$, but not with the presence of upper or lower motor neuron findings in the arms, or with autonomic dysfunction. Twenty-three of $25(92 \%)$ SHS patients with adequate follow up had satisfactory resolution of symptoms with conservative therapy (i.e. neither systemic corticosteroids nor stellate ganglion block), but only after a mean of 121 days (range 42-274 days).

Keywords: shoulder-hand syndrome; reflex sympathetic dystrophy; quadriplegia; spinal cord injury.

\section{Introduction}

Traumatic injury to the cervical spinal cord may cause quadriparesis or quadriplegia. Patients with such injuries generally require months of inpatient rehabilitation therapy directed towards maximizing function despite chronic deficits. Rehabilitation programs utilize intensive physical and occupational therapy techniques to improve patients' ability to use assistive devices, transfer between bed, wheelchair or walker and commode, and to maintain independence in activities of daily living. In such programs, patients are trained to use the upper extremities as the weight-bearing limbs of locomotion.

Upper extremity pain has long been recognized as a significant problem complicating the rehabilitation of cervical spinal cord injury patients. ${ }^{1}$ Nerve root compromise at the level of injury can produce radicular pain, while lesions of ascending spinal tracts may cause funicular pain, which is characteristically bilateral, diffuse and poorly localized, at or below the level of injury. ${ }^{2-4}$ Shoulder pain has been reported in about half of patients with spinal cord injury, and is usually attributed to overuse syndromes (e.g. tendinitis, bursitis, rotator cuff tear) resulting from vigorous rehabilitation therapy. ${ }^{5}$

We have observed that many cervical spinal cord injury patients with upper extremity pain have features of shoulderhand syndrome ${ }^{-}$(SHS), one of the syndromes encompassed by the term reflex sympathetic dystrophy (RSD). SHS has recently been emphasized as a common problem complicating the rehabilitation of stroke patients; ${ }^{6}$ the diagnosis is important because in this setting the syndrome responds well to specific therapy. 7,8 There are few reports, however, of SHS in spinal cord injury patients. Ohry et $a l^{9}$ briefly described two spinal cord injury patients with features of SHS. Gellman et al ${ }^{10}$ reviewed 60 consecutive spinal cord injury patients admitted for rehabilitation and found seven cases $(11.7 \%)$ of SHS. Three of these patients were treated with stellate ganglion blockage with good response. Cremer et al ${ }^{11}$ reported five patients with traumatic cervical spinal cord injury who developed SHS, three of whom improved after treatment with sys- 
temic steroids. One case of bilateral RSD was reported in a patient with traumatic central cord syndrome. ${ }^{12}$

We conducted a retrospective review of 43 consecutive patients with cervical spinal cord injury admitted to an inpatient rehabilitation center to determine the incidence and course of SHS.

\section{Patients and methods}

All patients admitted to the Burke Rehabilitation Center during a four year interval (1987-1991) were identified using a hospital database containing discharge diagnoses. This period was selected because all spinal cord injury patients during these years were under the care of one of the authors (MLA). The medical records of these patients were reviewed and abstracted, with attention to clinical features of SHS: pain, edema, vasomotor changes (instability of skin color and/or temperature), trophic changes (shiny, atrophic skin and/or dystrophic nails) and radiographic abnormalities. The progress notes of physicians, nurses and therapists were the primary sources of clinical data. X-rays were retrieved and reassessed.

To determine the relationship between manifestations of SHS and type of injury, patients were classified as having complete or incomplete cervical cord injury. Complete injury indicates no functional motor activity and no sensation below the level of injury. Patients with incomplete injuries were further subdivided into two groups: central cord injuries and noncentral cord injuries. Patients with central cord injuries have predominantly upper extremity weakness, and dissociated sensory loss with sparing of the posterior column pathways; typically, these are older patients with preexisting degenerative disease of the cervical spine.

Statistical analysis was performed using the SPSS/PC+ version 3.0 software package (SPSS Inc, Chicago, USA).

\section{Results}

The medical records of 43 patients (32 males, 11 females) admitted for rehabilita- tion following traumatic cervical spinal cord injury were studied. The mean age was 39 years, with a range of $16-86$. The mean time between injury and admission to the rehabilitation unit was 60 days, with a range of 5-187.

Twenty-four patients had complete cervical cord injuries. Among the 19 patients with incomplete injuries, 13 had central cord injuries. The mean age of the central cord injury patients was 59 years.

Features of SHS in the 43 patients are shown in Table I. Vasomotor and trophic changes may have been more prevalent than indicated, as these findings may be underreported in progress notes. Twenty-seven patients $(63 \%)$ had three or more features; we considered these to have probable SHS.

Among our patients with probable SHS, symptoms were bilateral in $24 / 27$ (89\%). Pain was usually worse in the weaker arm. The mean time from admission to onset of pain was 24 days; $14 / 27$ patients had onset of pain during the first week on the rehabilitation unit.

Shoulder pain was more common in patients with upper cervical (C3-5) injuries $(23 / 23,100 \%)$ than in patients with lower cervical injuries $(15 / 20,75 \%, p=0.02$ by Fisher's exact test). In contrast, hand/wrist pain occurred with the same frequency in patients with upper and lower cervical lesions.

Stepwise multiple regression analysis was used to assess the influence of the following factors on the number of SHS findings: age, sex, type of injury, the presence of upper or lower motor neuron findings in the upper extremities and autonomic dysfunction as indicated by orthostatic hypotension. Bivariate analyses revealed that age was the most significant factor $(r=0.495$,

Table I Features of SHS in 43 consecutive cervical cord injury patients

\begin{tabular}{lc}
\hline Shoulder pain & $38(88 \%)$ \\
Hand/wrist pain & $21(49 \%)$ \\
Edema & $21(49 \%)$ \\
Vasomotor changes & $19(44 \%)$ \\
Trophic changes & $6(14 \%)$ \\
Osteoporosis & $13 / 19$ \\
\hline
\end{tabular}


$p=0.0007)$. After adjusting for age, the only factor significantly predictive of number of SHS features was the presence of complete spinal cord injury $(p=0.03)$. Among patients with incomplete injuries, patients with the central cord pattern of injury had more features of SHS (mean 3.46) than those with noncentral cord injuries (mean 1.33), but this difference had no statistical significance after adjusting for age.

X-rays had been obtained to evaluate shoulder or hand pain in 19 patients. The characteristic radiographic finding was osteoporosis, which was occasionally severe, with cyst-like changes in the hands and wrists (Fig 1) or humeral heads. One patient had heterotopic ossification of the shoulder. Bone scans, unavailable at our rehabilitation center, were not obtained.

All patients were treated with daily intensive physical and occupational therapy, including heat modalities and range of motion exercises. All received nonsteroidal antiinflammatory drugs, and all used their hands, wrists, elbows and shoulders as weight bearing joints. Treatment with systemic corticosteroids and stellate ganglion block was only considered after the failure of more conservative therapy over many months, as noted below. Of note, 15 patients were treated with phenoxybenzamine (for neurogenic bladder symptoms) without influence on severity or incidence of SHS features. Of 27 patients with probable SHS, adequate follow up was available for 25 (one

a

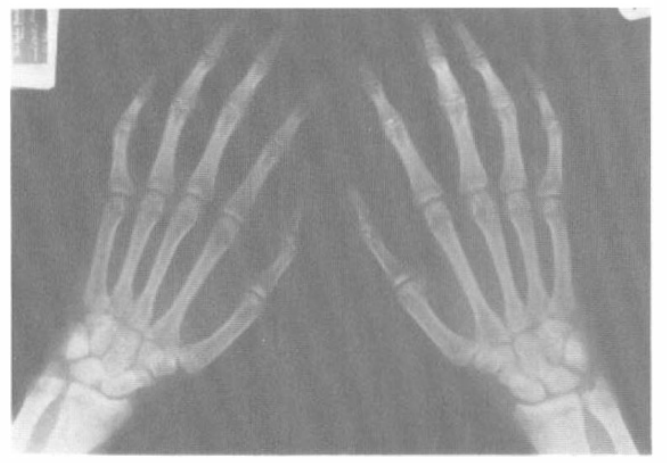

patient was transferred to another institution and one signed out prematurely against medical advice). Twenty-three of 25 patients had satisfactory resolution of pain, after a mean of 121 days (range 42-274 days) from the onset of symptoms.

Two patients continue to suffer from severe, diffuse burning pain of all extremities, perhaps a form of generalized reflex sympathetic dystrophy, over a year after the onset of symptoms. Both have central cord syndrome and are over 60 years old. One has had only transient benefit from systemic corticosteroids and stellate ganglion blocks, and continues to require oral narcotic analgesics. The other patient who failed to improve did not respond to treatment with carbamazepine, clonazepam, baclofen, mexiletine, amitriptyline and biofeedback.

Shoulder-hand syndrome appeared to delay the attainment of rehabilitation goals, and may have influenced the length of stay on the rehabilitation unit. As expected, the length of stay correlated most strongly with the presence of complete cord injury ( $r=0.473, p=0.0014)$. Among patients with complete injuries, there was a trend towards an association between the number of SHS features and length of stay $(r=0.309, p=0.07)$.

\section{Discussion}

Pain is a common, well known complication of cervical spinal cord injury; it is often a major impediment to the rehabilitation pro-

b

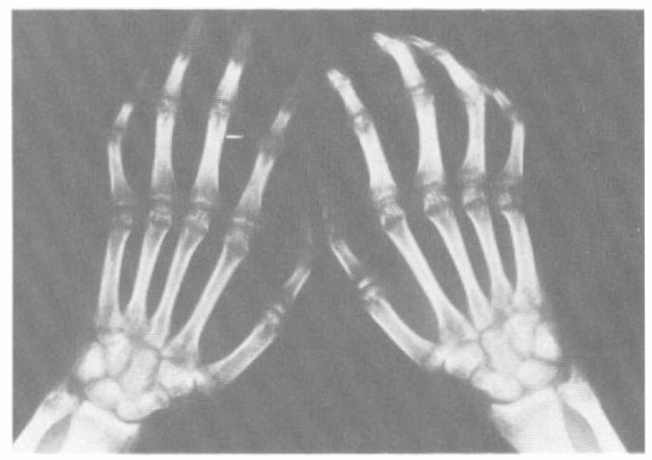

Figure 1 Hand radiographs of a patient with shoulder-hand syndrome demonstrating the progression of severe osteoporosis. (a) 2 months after injury. (b) 4 months after injury. 
gram. Pain has most often been considered a symptom of overuse, but has also been attributed to radicular or funicular damage at the level of injury. However we have been impressed by the frequency with which findings associated with RSD, specifically SHS, ocur in these patients: poorly localized burning pain, diffuse edema, vasomotor changes, thin shiny skin, dystrophic nails and osteoporosis on $\mathrm{x}$-ray. It may be argued that some of these features, particularly edema and osteoporosis, can result from disuse alone. However, all of the patients studied participated in vigorous upper extremity exercise programs, using shoulders, elbows, wrists and hands as weight bearing supports during transfers and to propel manual wheelchairs. We do not believe that the constellation of signs and symptoms present in this group of spinal cord injury patients can be explained on the basis of overuse syndromes and disuse atrophy.

Kozin et al $^{13}$ proposed diagnostic criteria for RSD. They considered definite RSD to consist of pain and tenderness of an extremity with vasomotor instability and swelling. Probable RSD was defined as pain and tenderness with vasomotor instability or swelling. Possible RSD included patients with vasomotor instability and swelling, but no pain and tenderness. Applying these criteria to our 43 spinal cord injury patients, eight $(19 \%)$ had definite RSD, $10(23 \%)$ had probable RSD and 11 (26\%) had possible RSD. The radiographic abnormalities of the hands and shoulders of some of our patients, including cyst-like erosions, are similar to those reported in RSD. ${ }^{14}$ Kozin et al found that 11 consecutive patients with RSD had bilateral involvement, suggesting a central neural mechanism. ${ }^{15}$ Symptoms were bilateral in the majority of our patients.

The frequency and distribution of SHS features in our spinal cord injury patients suggests that there may be a continuum from simple loss of function due to disuse to full-blown RSD. The clinical findings associated with increased risk of SHS manifestations were age and complete cord injury. Interestingly, lower motor neuron signs, degree of generalized autonomic dysfunction (orthostatic hypotension, autonomic hyperreflexia, neurogenic bowel and bladder), and medication with the alphaadrenergic receptor blocker phenoxybenzamine, did not influence development of SHS.

Accurate classification may have important therapeutic implications. Though most of these patients had a satisfactory outcome, the mean time to resolution of symptoms was 4 months, greatly retarding rehabilitation. If the symptom duration can be shortened with specific therapeutic measures, such as systemic corticosteroids, the gains may be considerable. Corticosteroid therapy has substantial benefit in SHS complicating stroke..$^{7,8}$

The pattern and frequency of SHS in cervical spinal cord injury also has interesting implications for the pathogenesis of $\mathrm{RSD}$. The relative importance of peripheral sensitization of receptors and abnormal spinal cord response has been a matter of debate (as recently reviewed by Schwartz$\operatorname{man}^{16}$ ). The fact that SHS and other forms of RSD occur in a wide variety of clinical settings, ${ }^{17}$ including injury to an extremity without apparent nerve damage, indicate that direct injury to the nervous system is not essential. But our data suggest that the highest incidence of RSD is in the setting of traumatic cervical cord injury. Cord damage with interruption of central regulatory influences may render these patients susceptible to pathological sympathetic response and pain. The correlation of SHS features with age suggests that age-related central and/or peripheral nervous system changes may be important.

Because this was a retrospective study, the clinical data available for analysis were incomplete. The results of careful assessment of quality and severity of pain, degree of swelling, vasomotor and trophic changes, and $\mathrm{x}$-rays were not available for all patients. Despite this limitation, the study demonstrates that features of SHS are very common in the setting of cervical spinal cord injury, raising two important opportunities. First, study of the efficacy of specific treatment modalities directed toward SHS in this setting may lead to important benefits in the rehabilitation of these patients. A trial of systemic corticosteroid therapy is currently 
under way at our institution. Second, careful prospective neurophysiologic study of these patients, from the time of injury through the course of SHS, may improve our understanding of the pathophysiology of this elusive disorder.

\section{References}

1 Davis L, Martin J (1947) Studies upon spinal cord injuries. II. The nature and treatment of pain. J Neurosurg 4: 483-491.

2 Davidoff G, Roth E, Guarrachini M et al (1987) Functional-limiting dysesthetic pain syndrome among traumatic spinal cord injury patients: a cross-sectional study. Pain 29: 39-48.

3 Nepomuceno C, Fine PR, Richards JS et al (1979) Pain in patients with spinal cord injury. Arch Phys Med Rehabil 60: 605-609.

4 Kaplan LI, Grynbaum BB, Floyd KE, Rusk HA (1962) Pain and spasticity in patients with spinal cord dysfunction: results of a follow-up study. JAMA 182: 918-924.

5 Nichols PJ, Norman P, Ennis JK (1979) Wheelchair users' shoulder pain in patients with spinal cord lesions. Scand J Rehabil Med 11: 29-32.

6 Chelson GG, Fitzpatrick KA, Navia RA et al (1987) Prevalence of the shoulder-hand syndrome in an inpatient stroke rehabilitation population: a quantitative cross-sectional study. J Neurorehabil 1: 137-141.

7 Davis SW, Petrillo CR, Eichberg RD, Chu DS (1977) Shoulder-hand syndrome in a hemiplegic population: a 5-year retrospective study. Arch Phys Med Rehabil 58: 353-356.

8 Braus DF, Strobel J (1991) The shoulder-hand syndrome after stroke: a prospective study. Ann Neurol 30: 278-279. (Abstract).

9 Ohry A, Brooks ME, Steinbach TV, Rozin R (1978) Shoulder complications as a cause of delay in rehabilitation of spinal cord injured patients. Paraplegia 16: 310-316. (Abstract).

10 Gellman H, Eckert RR, Botte MJ et al (1988) Reflex sympathetic dystrophy in cervical spinal cord injury patients. Clin Orthop 233: 126-131.

11 Cremer SA, Maynard F, Davidoff G (1989) The reflex sympathetic dystrophy syndrome associated with traumatic myelopathy: report of 5 cases. Pain 37: 187-192.

12 Philip PA, Philip M, Monga TN (1990) Reflex sympathetic dystrophy in central cord syndrome: case report and review of the literature. Paraplegia 28: 48-54.

13 Kozin F, Ryan LM, Careerra GF et al (1981) The reflex sympathetic dystrophy syndrome (RSDS). III. Scintigraphic studies, further evidence for the therapeutic efficacy of systemic corticosteroids, and proposed diagnostic criteria. Am J Med 70: 23-30.

14 Kozin F, McCarty DJ, Sims J, Genant HK (1976) The reflex sympathetic dystrophy syndrome. I. Clinical and histologic studies: evidence for bilaterality, response to corticosteroids, and articular involvement. Am J Med 60: 321-331.

15 Kozin F, Genant HK, Bekerman C, McCarty DJ (1976) The reflex sympathetic dystrophy syndrome. II. Roentgenographic and scintigraphic evidence of bilaterality and of periarticular accentuation. Am J Med 60: 332-338.

16 Schwartzman RJ (1992) Reflex sympathetic dystrophy and causalgia. Neurol Clin 10: 953-973.

17 Steinbrocker O, Argyros TG (1958) The shoulder-hand syndrome: present status as a diagnostic and therapeutic entity. Med Clin N Am 42: 1533-1553. 